\title{
Experiencia con Ecocardiograma de estrés dipiridamol y Reserva coronaria no invasiva. Comunicación preliminar
}

\author{
Mario Alfaro ${ }^{1}$, Gerardo Palma ${ }^{1}$, Óscar Moreno ${ }^{1}$, Mariela Miranda ${ }^{2}$, Mariluz Medina ${ }^{3}$, \\ Belén Abara. ${ }^{3}$
}

1. Centro Cardio-Vascular, Hospital San Borja Arriarán, Santiago, Chile.
2. Enfermera Universitaria CDT San Borja Arriarán, Santiago, Chile.
3. Internas de Medicina, Santiago Centro, Universidad de Chile

Antecedentes: El Ecocardiograma de Estrés (EE) es una técnica ampliamente utilizada en el estudio de pacientes con Enfermedad Coronaria conocida o sospechada. Recientemente se ha preconizado efectuar el test vasodilatador, método recomendado para evaluar no invasivamente la reserva de flujo coronario (RFC).

Objetivos: Mostrar la experiencia preliminar con el EE Dipiridamol, con un seguimiento inicial, así como el estudio de la RFC en la porción distal de la arteria Descendente Anterior (ADA).

Métodos: Se analizaron 164 pacientes (101 hombres, edad promedio 65 años) a los que se les efectuó un EE Dipiridamol entre Julio 2011 y Septiembre 2012, con una dosis de $0.84 \mathrm{mg} / \mathrm{kg}$ en 6 minutos, que incluyó el estudio de RFC. Todos los pacientes completaron la prueba sin efectos secundarios significativos, y en un tiempo menor a 25 minutos. El seguimiento se hizo con miras a la aparición de eventos cardiovasculares mayores (revascularización, infarto al miocardio y muerte cardíaca). Resultados: EE resultó positivo para isquemia en
$19(21 \%)$ pacientes. En 19 (12\%) el EE resultó alterado por motilidad parietal; $29(15 \%)$ presentaron respuesta anormal en la RFC, con una coincidencia de 12 pacientes (7\%) con ambos tipo de alteración; En 142 (88\%) pacientes se logró medir la RFC en ADA. En 151 pacientes se obtuvo datos confiables del seguimiento, que fue en promedio 10 meses ( 6 a 18); entre estos, 10 pacientes tuvieron alguna revascularización. No hubo infartos ni fallecidos por causa cardio-vascular. Dado el corto período de seguimiento y baja incidencia de eventos, no se pudo establecer el valor predictivo del resultado de la prueba en esta muestra.

Conclusiones: Esta experiencia permitió familiarizarse con la técnica del EE con Dipiridamol y estudiar en un alto porcentaje la RFC en la ADA. La técnica es segura y consume muy poco tiempo. Queda pendiente la determinación del valor predictivo de estos resultados en nuestras manos.

Palabras claves: Eco estrés, dipiridamol, reserva de flujo coronario, estudio no invasivo. 


\section{Dipyridamol Stress Echocardiography and Non-invasive Coronary Reserve. Preliminary report.}

Background: Stress Echocardiography is a common technique in the study of patients with known or suspected Coronary artery disease (CAD). Recently the vasodilation test has been recommended for the non invasive study of Coronary Flow Reserve (CFR).

Aim: to report our preliminary experience with Dipyridamol Stress Echocardiography (DSE), an initial follow-up, and the study of CFR in the distal portion of the left anterior descending coronary artery (LAD).

Methods: 164 consecutive patients (101 men, mean age 65) with suspected or known CAD underwent a high-dose DSE $(0.84 \mathrm{mg} / \mathrm{kg}$ over $6 \mathrm{~min})$ between July 2011 and September 2012. The test included a study of CFR.All patients completed the test within $25 \mathrm{~min}$. Prospective follow-up regarding major adverse cardiovascular events(revascularization, cardiac mortality and infarction) was conducted for a mean of 10 months (range 6-18)

Results: DSE was positive for ischemia in 34 (21\%) patients, showed abnormal wall motion in 19 (12\%), abnormal CFR in 29 (15\%), and both abnormalities in 12 (7\%). 142 patients (88\%) had a normal CFR in the LAD. 151 patients were followed for a mean of 10 months ( 6 to 18 ). Ten of these patients required some type of revascularization. There were no myocardial infarctions or cardiac deaths. Due to the short follow up period and the low incidence of events, it was not possible to estimate the predictive value of abnormal CFR. Conclusion: The dipyridamol echo stress test with a study of coronary flow reserve is safe and brief. The predictive value for cardiac events remains to be determined.

Keywords: Dipirydamol, Stress echocardiography, coronary flow reserve, non invasive test

\section{Introducción}

El Ecocardiograma de Estrés (EE) es una técnica muy utilizada en el estudio de pacientes con Enfermedad Coronaria conocida o sospechada. Ha sido ampliamente demostrado que en pacientes con Enfermedad Coronaria, el EE farmacológico o de ejercicio es un predictor independiente de muerte, sumándose a otros parámetros clínicos y ecocardiográficos ${ }^{1-3}$.

La Dobutamina ha sido el fármaco más usado, inótropo positivo, que actúa por aumento del consumo de oxígeno miocárdico. En el último tiempo se ha incorporado el Dipiridamol, fármaco vasodilatador indirecto, que genera robo de flujo en la circulación coronaria; éste además per- mite evaluar no invasivamente la reserva de flujo coronario (RFC), por lo que hoy la combinación del análisis convencional de la motilidad, mediante la ecocardiografía bidimensional, y el Doppler en la porción medio-distal de la arteria Descendente Anterior (ADA), es la técnica recomendada durante el $\mathrm{EE}$ con vasodilatador ${ }^{4}$.

Diversos autores han publicado resultados comparables en cuanto a rendimiento diagnóstico, valores pronósticos y predictivos, entre los estudios de EE con Dobutamina y Dipiridamol ${ }^{5}$. Más recientemente, trabajos demuestran que el estudio de la RFC le da mayor valor pronóstico al EE, por sobre el resultado en términos de isquemia parietal, en varios tipos de pacientes: Enfermedad Coronaria 
conocida o sospechada ${ }^{6,7}$, Diabetes Mellitus ${ }^{8}$, arterias coronarias normales ${ }^{9}$, estratificación de dolor torácico, tanto en hombres como en mujeres. Además la RFC no invasiva es independiente del resultado de la respuesta de motilidad parietal $^{10}, \mathrm{y}$ no es influido por la presencia de terapia médica que sí afecta la motilidad parietal ${ }^{11}$.

En un estudio a gran escala de reciente publicación, con más de 4000 pacientes seguidos por un promedio de 19 meses, la RFC anormal resultó un potente predictor de mortalidad, donde una tasa de 5.2 para isquemia parietal sola, aumenta a 17.6 con la combinación de isquemia/ RFC anormal ${ }^{12}$.

Esta medición no invasiva de la reserva coronaria ha sido también comparada con la reserva de flujo fraccional (FFR) efectuada con guía de presión durante el estudio angiográfico, en el escenario de lesiones intermedias, que justamente plantean el desafío de definir la significación funcional. En una publicación de 50 casos, con ambos estudios, la concordancia fue $88 \%$, si bien en este caso se usó Adenosina intravenosa directa ${ }^{13}$.

El propósito de esta publicación es dar a conocer la experiencia de nuestro Centro, con algo más de un año utilizando el EE dipiridamol en el estudio de isquemia miocárdica y en la estimación de la reserva de flujo coronario.

\section{Pacientes y Métodos}

Se enrolaron 162 pacientes consecutivos (101 hombres, edad promedio 65 años) que fueron derivados para un $\mathrm{EE}$ en estudio de isquemia, a la Unidad de Ecocardiografía del Centro Cardio-Vascular del Hospital San Borja. Las características demográficas y clínicas de los pacientes se describen en la Tabla 1.

\begin{tabular}{|c|c|}
\hline Características de los pacientes & $(\%)$ \\
\hline $\begin{array}{l}\text { Edad promedio (años) } \\
\text { Sexo masculino }\end{array}$ & $\begin{array}{l}65 \\
101(62)\end{array}$ \\
\hline $\begin{array}{l}\text { Hipertensión Arterial } \\
\text { Enf. Coronaria } \\
\text { Angioplastía } \\
\text { Cirugía Revascularización }\end{array}$ & $\begin{array}{l}110(68) \\
43(27) \\
22(14) \\
10(6)\end{array}$ \\
\hline $\begin{array}{l}\text { Probabilidad pre-test } \\
\text { Baja } \\
\text { Media } \\
\text { Alta }\end{array}$ & $\begin{array}{l}78(42) \\
30(25) \\
53(33)\end{array}$ \\
\hline
\end{tabular}

Todos los pacientes, luego de firmar el consentimiento informado institucional, fueron sometidos al examen de EE con Dipiridamol, de acuerdo al protocolo propuesto por la Asociación Europea de Ecocardiografía ${ }^{1}$, recibiendo una dosis de $0.84 \mathrm{mg} / \mathrm{kg}$ en infusión directa en 6 minutos. Se les efectuó un Ecocardiograma Doppler basal completo; se adquirieron imágenes ecocardiográficas de la motilidad parietal en vistas de 4, 2 y 3 cámaras apical y el estudio de Doppler en la porción distal de la ADA, según técnica descrita $^{14,15}$. Se monitoreó electrocardiograma de 3 derivaciones y la presión arterial cada 3 minutos. Se adquirieron nuevas imágenes de motilidad parietal en las mismas vistas al término de la infusión (6 minutos), el efecto máximo (minuto 10-12) y tras revertir el efecto vasodilatador con Aminofilina en dosis de $125 \mathrm{mg}$. intravenoso directo. El flujo de la ADA se midió nuevamente durante el período de efecto vasodilatador máximo. Todos los exámenes fueron efectuados con un mismo equipo, General Electric Vivid 7®. Los exámenes fueron almacenados digitalmente en formato que permite su posterior revisión y medición (DVD-Dicom ${ }^{\circledR}$ ).

El análisis e informe de la motilidad parietal se efectuó de acuerdo a las recomendaciones internacionales ${ }^{16}$, con la segmentación del Ventrículo Izquierdo incluida en el ecocardiógrafo (16 segmentos); la velocidad del flujo de la ADA (promedio de 3 mediciones en el punto máximo de la señal espectral) se comparó entre la medición en vasodilatación máxima y la basal, informándose como una RFC normal cuando el cociente fue mayor o igual a 2. En las Figuras 1 a 3 se muestran ejemplos de la imagen de la ADA en el Doppler-color, y las mediciones de la velocidad de flujo, basal y en vasodilatación.

Los pacientes fueron categorizados según la probabilidad pre-tes, $\mathrm{t}$ alta, media o baja, de acuerdo a las recomendaciones de la American Society of Echocardiography 17.

Para el seguimiento, se revisaron las fichas clínicas de los pacientes, así como los registros internos de las Unidades de Angiografía y Cardio-cirugía, y contacto telefónico, con miras a establecer la incidencia de eventos mayores cardio-vasculares, revascularización quirúrgica o percutánea, infarto al miocardio o muerte cardíaca.

\section{Resultados}

Los 162 pacientes completaron la prueba satisfactoriamente. En 142 pacientes (88\%) se lograron imágenes ade- 


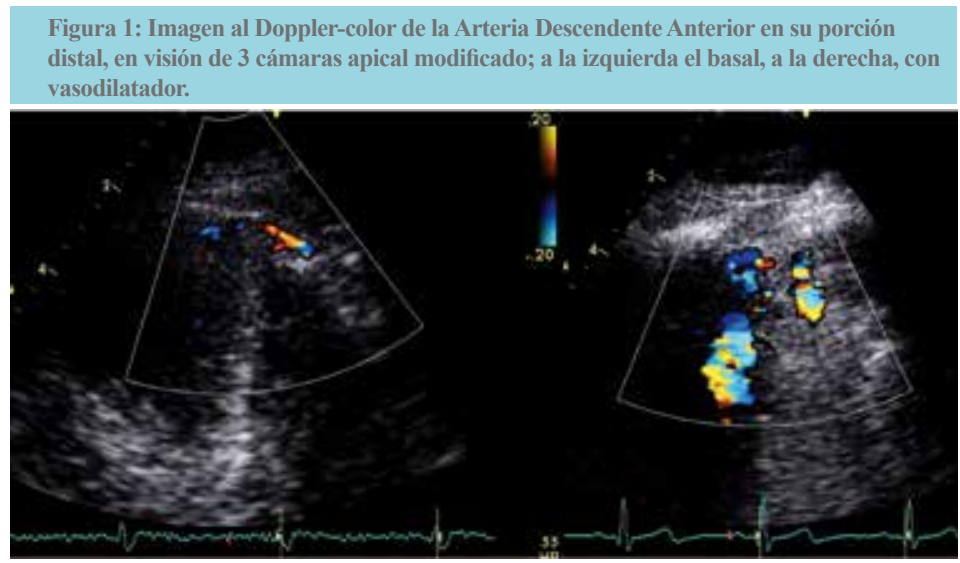

\section{Figura 2: Curva espectral de Doppler Pulsado en la ADA distal, registro basal} (izquierda) y a derecha, respuesta normal de aumento del flujo $>$ a 2 veces.

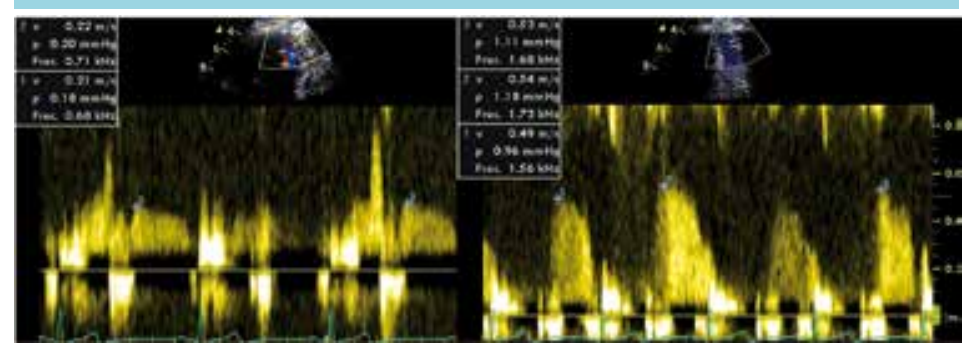

Figura 3: Curva espectral de Doppler Pulsado de ADA distal, con registro basal (izquierda) y registro con vasodilatación y respuesta anormal, aumento del flujo $<$ a 2 veces.

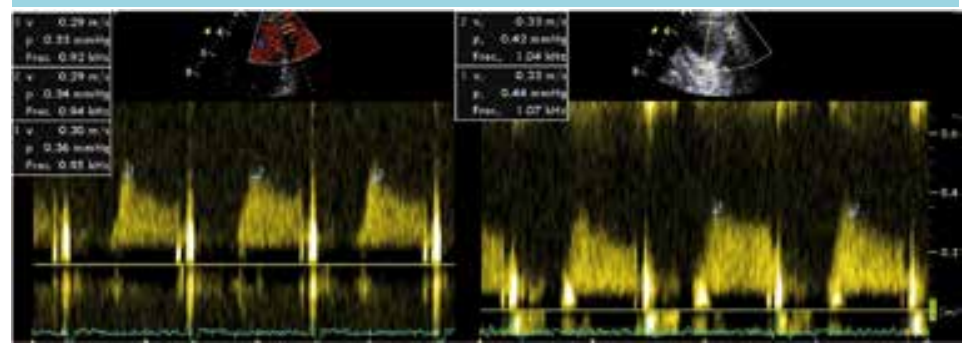

cuadas y registro confiable del Doppler de la ADA, tanto basal como en vasodilatación, para establecer la respuesta de la RFC. La prueba se efectuó en un lapso de 20 a 25 minutos, al tener solamente 2 etapas de adquisición de imágenes en estrés, más una de recuperación; hubo síntomas generales atribuibles al efecto del vasodilatador, cefalea y sensación de bochorno facial, que fueron discretos y en ningún caso se debió suspender la infusión del fármaco Un total de 34 pacientes tuvieron una prueba alterada, correspondiente al $21 \%$ del total de la muestra. En 19 pa- cientes (12\%) el EE resultó alterado por isquemia parietal; 29 pacientes (15\%) presentaron respuesta anormal en la RFC, con una coincidencia de 12 pacientes (7\%) con ambas alteraciones. 128 pacientes tuvieron resultado normal para el EE y la RFC (79\%).

Un tercio de los pacientes (53) fueron categorizados con probabilidad pre-test alta, y en estos, la positividad de isquemia parietal sube a $32 \%$, y para la RFC anormal, a $42 \%$.

El seguimiento promedio fue de 10 meses (rango 6 a 18); 
en 13 pacientes no se tuvo información confiable de su evolución, todos del grupo con resultados normales; 3 pacientes fallecieron durante el período, correspondientes al grupo sin alteraciones de la prueba de EE, y la causa del deceso no se atribuyó a Enfermedad Cardiaca.

En el grupo de pacientes con EE isquémico y RFC anormal, 6 de 12 pacientes presentaron eventos, incluidos Infarto al Miocardio, Angioplastía Coronaria y/o Cirugía de Revascularización Miocárdica.

Entre los 5 pacientes con EE negativo y RFC anormal, los eventos fueron 2. En el grupo de 17 pacientes con EE positivo y RFC anormal ocurrió 1 evento. En el grupo de ambos resultados normales, EE (-) y RFC (-), hubo 1 evento, Cirugía de revascularización miocárdica en un paciente joven con anomalía de nacimiento de Arteria Coronaria Circunfleja (Tabla 2).

\begin{tabular}{|c|c|c|}
\hline \multicolumn{3}{|c|}{ Tabla 2: Eventos durante el seguimiento } \\
\hline $\begin{array}{l}\text { Resultado de } \\
\text { la prueba }\end{array}$ & $\begin{array}{r}\text { Eventos } \\
\text { Mayores }\end{array}$ & $\begin{array}{c}\text { Muerte } \\
\text { cardiovascular }\end{array}$ \\
\hline $\begin{array}{ll}\mathrm{EE}+\text { y RFC + } & (12) \\
\mathrm{EE}+\text { y RFC (-) } & (5) \\
\mathrm{EE}(-) \text { y RFC + } & (17) \\
\text { EE }(-) \text { y RFC (-) } & (114)\end{array}$ & $\begin{array}{l}6 \\
2 \\
1 \\
1\end{array}$ & $\begin{array}{l}\mathbf{0} \\
\mathbf{0} \\
\mathbf{0} \\
\mathbf{0}\end{array}$ \\
\hline \multicolumn{3}{|c|}{$\begin{array}{l}\text { EE +: prueba positiva para isquemia parietal } \\
\text { EE (-): prueba negativa para isquemia parietal } \\
R F C+\text { : reserva coronaria anormal } \\
R F C(-) \text { : reserva coronaria normal. }\end{array}$} \\
\hline
\end{tabular}

\section{Discusión}

En esta comunicación mostramos la experiencia inicial con la técnica del EE con Dipiridamol, única en el país según nuestra información. En un alto porcentaje de pacientes (88\%), se obtiene el estudio de la Reserva de Flujo Coronario; entre las causas de la falla en lograrlo se incluyen pacientes con ventana ultrasónica no propicia y pacientes con arteria Descendente Anterior ocluida en evento coronario previo.

Los resultados en términos de pruebas anormales resultan inferiores a los publicados en series internacionales, posiblemente explicable por tratarse de una muestra no seleccionada de pacientes, con predomino de pacientes con baja y probabilidad pre-test; de hecho, al separar los resultados según esta probabilidad, la positividad de la prueba es mucho mayor.

En la numerosa serie de Cortigiani y Rigo, la positividad es de $18 \%$ para isquemia parietal y $33 \%$ para la reserva anormal, con mortalidad a 4 años marcadamente mayor en el grupo con ambas alteraciones, pero especialmente en el análisis multivariado fue la RFC el mejor predictor ${ }^{12}$.

El seguimiento de nuestros pacientes es aún breve, lo que puede explicar que se registren escasos eventos, salvo en el grupo que tiene alteración de ambos componentes de la prueba $(50 \%)$.

En la serie de Wejner-Mik, Liepec y Kasprzak, un 54\% de los pacientes tuvieron eventos en un seguimiento medio de $32 \pm 11$ meses, con alta predicción por la presencia de cualquier anomalía inducible; pero un $75 \%$ de los pacientes tenían lesiones coronarias $\geq 70 \%{ }^{18}$.

Una observación más prolongada, y la incorporación de mayor número de pacientes, nos permitirá establecer la información estadística en cuanto al valor predictivo de este procedimiento en nuestras manos.

Otros elemento destacable de esta experiencia es la ausencia de manifestaciones adversas significativas; el empleo de Dipiridamol como fármaco apremiante tiene muy pocas contraindicaciones, entre las cuales están los bloqueos aurículo-ventriculares y Asma Bronquial descompensada, no habiendo problema de usarlo en pacientes con Hipertensión Arterial mal controlada, arritmias supraventriculares y ventriculares ${ }^{4}$.

Destacable es el tiempo que consume la realización de la prueba, aproximadamente la mitad de una prueba convencional de EE con Dobutamina, lo que resulta en otra ventaja operativa y un factor importante en nuestro trabajo cotidiano.

Finalmente, nos hemos limitado a los estudios de isquemia, dejando fuera estudios de viabilidad, para lo cual seguimos empleando el protocolo de bajas dosis con Dobutamina, según recomendaciones ${ }^{4,16}$.

En conclusión, con esta técnica del EE con Dipiridamol, se obtiene el estudio de RFC en un alto porcentaje de pacientes. La técnica es segura, tiene muy pocas limitaciones en la práctica, y consume menos tiempo que la prueba con Dobutamina. Queda pendiente la determinación del valor predictivo de estos resultados en nuestra experiencia. 


\section{Referencias:}

1. MARWICK TH, CASE C, SAWADA S, RIMMERMAN C, BRENNEMAN P, KOVACS R, et al. Prediction of mortality using dobutamine echocardiography. J Am Coll Cardiol 2001;37:754-60

2. MARWICK TH, CASE C, VASEY C, ALLEN S, SHORT L, THOMAS JD. Prediction of mortality by exercise echocardiography. Circulation 2001;103:2566-71.

3. SICARI R, PASANISI E, VENNERI L, LANDI P, CORTIGIANI L, PICANO E. Stress echo results predict mortality: a large-scale multicenter prospective international study. J Am Coll Cardiol 2003;41: 589-95.

4. SICARI R, NIHOYANNOPOULOS P, EVANGELISTA A, KASPRZAK J, LANCELLOTTI P, POLDERMANS D, et al. Stress echocardiography expert consensus statement: the European Association of Echocardiography (EAE) (a registered branch of the ESC). Eur J Echocardiogr 2008; 9:415-37.

5. PICANO E, MOLINARO S, PASANISI E. The diagnostic accuracy of pharmacological stress echocardiography for the assessment of coronary artery disease: a meta-analysis. Cardiovasc Ultrasound 2008;6:30

6. RIGO F, SICARI R, GHERARDI S, DJORDJEVIC-DIKIC A, CORTIGIANI L, PICANO E. The additive prognostic value of wall motion abnormalities and coronary flow reserve during dipyridamole stress echo. Eur Heart J 2008;29: 79-88.

7. CORTIGIANI L, RIGO F, GALDERISI M, GHERARDI S, BOVENZI F, PICANO E, et al. Diagnostic and prognostic value of Doppler echocardiographic coronary flow reserve in the left anterior descending artery. Heart 2011; 97:1758 - 65 .

8. CORTIGIANI L, RIGO F, GHERARDI S, SICARI R, GALDERISI M, BOVENZI F, et al. Additional prognostic value of coronary flow reserve in diabetic and nondiabetic patients with negative dipyridamole stress echocardiography by wall motion criteria. J Am Coll Cardiol 2007;50:1354-61

9. SICARI R, RIGO F, CORTIGIANI L, GHERARDI S, GALDERISI M, PICANO E. Additive prognostic value of coronary flow reserve in patients with chest pain syndrome and normal or near-normal coronary arteries. Am J Cardiol 2009; 103:626-31.
10. CORTIGIANI L, RIGO F, GHERARDI S, GALDERISI M, BOVENZI F, PICANO E, et al. Prognostic effect of coronary flow reserve in women versus men with chest pain syndrome and normal dipyridamole stress echocardiography. Am J Cardiol 2010;106:1703-8.

11. SICARI R, RIGO F, GHERARDI S, GALDERISI M, CORTIGIANI L, PICANO E. The prognostic value of Doppler echocardiographic-derived coronary flow reserve is not affected by concomitant antiischemic therapy at the time of testing. Am Heart J 2008;156: 573-9.

12. CORTIGIANI L, RIGO F, GHERARDI S, BOVENZI F, MOLINARO S, PICANO E, et al. Coronary Flow Reserve during Dipiridamole Stress Echocardiography predicts mortality. J. Am Coll Cardiol Img 2012; 5: 1079-85.

13. MEIMOUN P, SAYAH S, LUYCX-BORE A, BOULANGER J, ELMKIES F, BENALI T, et al. Comparison between NonInvasive Coronary Flow Reserve and Fractional Flow Reserve to assess the Funcional Significance of Left Anterior Descending Artery Stenosis of Intermediate Severity. J Am Soc Echocardiogr 2011; 24: 374-81.

14. LOWENSTEIN J, TIANO C, MANSO H, PELLEGRINI C. Determinación de la reserva coronaria por eco-Doppler transtorácico. Rev Argentina de Cardiología 2000; 68: 383-98.

15. VOCI P, PIZZUTO F, ROMEO F. Coronary flow: a new asset for the echo lab. Eur Heart J 2004; 25: 1867-79.

16. ARMSTRONG WF, PELLIKA PA, RYAN T, CRUSE L, ZOGHBI W. Stress Echocardiography Task Force of the nomenclature and Standards Committee of the American Society of Echocardiography. Stress Echocardiography recommendations for performance and interpretation of stress echocardiography. $\mathrm{J}$ Am Soc Echocardiogr 1998;11:97-104.

17. DOUGLAS PS, KHANDERIA B, STAINBACK RF, WEISSMAN NJ, PETERSON ED, HENDEL RC, et al. 2008 appropriateness criteria for stress echocardiography. a report of ACCF/ASE/ACEP/AHA/ASNC/SCAI/ACCT/SCMR. J Am Coll Cardiol 2008;51: 1127-47.

18.- WEJNER-MIK P, LIPIEC P, KASPRZAK JD. Long-term prognostic value of dipyridamole stress myocardila contrast echocardiography. Eur J Echocardiogr 2011; 12:762-66 